ERNEST DRLANDD LAWRENCE BERKELEY NATIONAL LABDRATIRY

The First Infrared Beamline at the ALS: Design, Construction, and Initial Commissioning

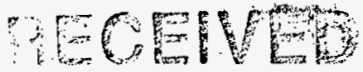

1.0V 001997

OSTI

W.R. McKinney, C.J. Hirschmugl,

H.A. Padmore, T. Lauritzen, N. Andresen, G. Andronaco, R. Patton, and M. Fong Accelerator and Fusion Research Division

September 1997

Presented at the SPIE Optical Science, Engineering and Instrumentation Conference, 1997, San Diego, CA, July 27-August 1, 1997, and to be published in the Proceedings

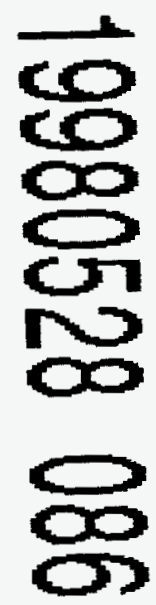

DTIC QUALTTY INOPECTED 1 


\section{DISCLAIMER}

This document was prepared as an account of work sponsored by the United States Government. While this document is believed to contain correct information, neither the United States Government nor any agency thereof, nor The Regents of the University of California, nor any of their employees, makes any warranty, express or implied, or assumes any legal responsibility for the accuracy, completeness, or usefulness of any information, apparatus, product, or process disclosed, or represents that its use would not infringe privately owned rights. Reference herein to any specific commercial product, process, or service by its trade name, trademark, manufacturer, or otherwise, does not necessarily constitute or imply its endorsement, recommendation, or favoring by the United States Government or any agency thereof, or The Regents of the University of California. The views and opinions of authors expressed herein do not necessarily state or reflect those of the United States Government or any agency thereof, or The Regents of the University of California.

Ernest Orlando Lawrence Berkeley National Laboratory is an equal opportunity employer. 


\title{
THE FIRST INFRARED BEAMLINE AT THE ALS: DESIGN, CONSTRUCTION, AND INITIAL COMMISSIONING
}

\author{
W.R. McKinney, C.J. Hirschmugl, H.A. Padmore, T. Lauritzen, \\ N. Andresen, G. Andronaco, R. Patton, M. Fong \\ Advanced Light Source \\ Ernest Orlando Lawrence Berkeley National Laboratory \\ University of California, Berkeley, California 94720
}




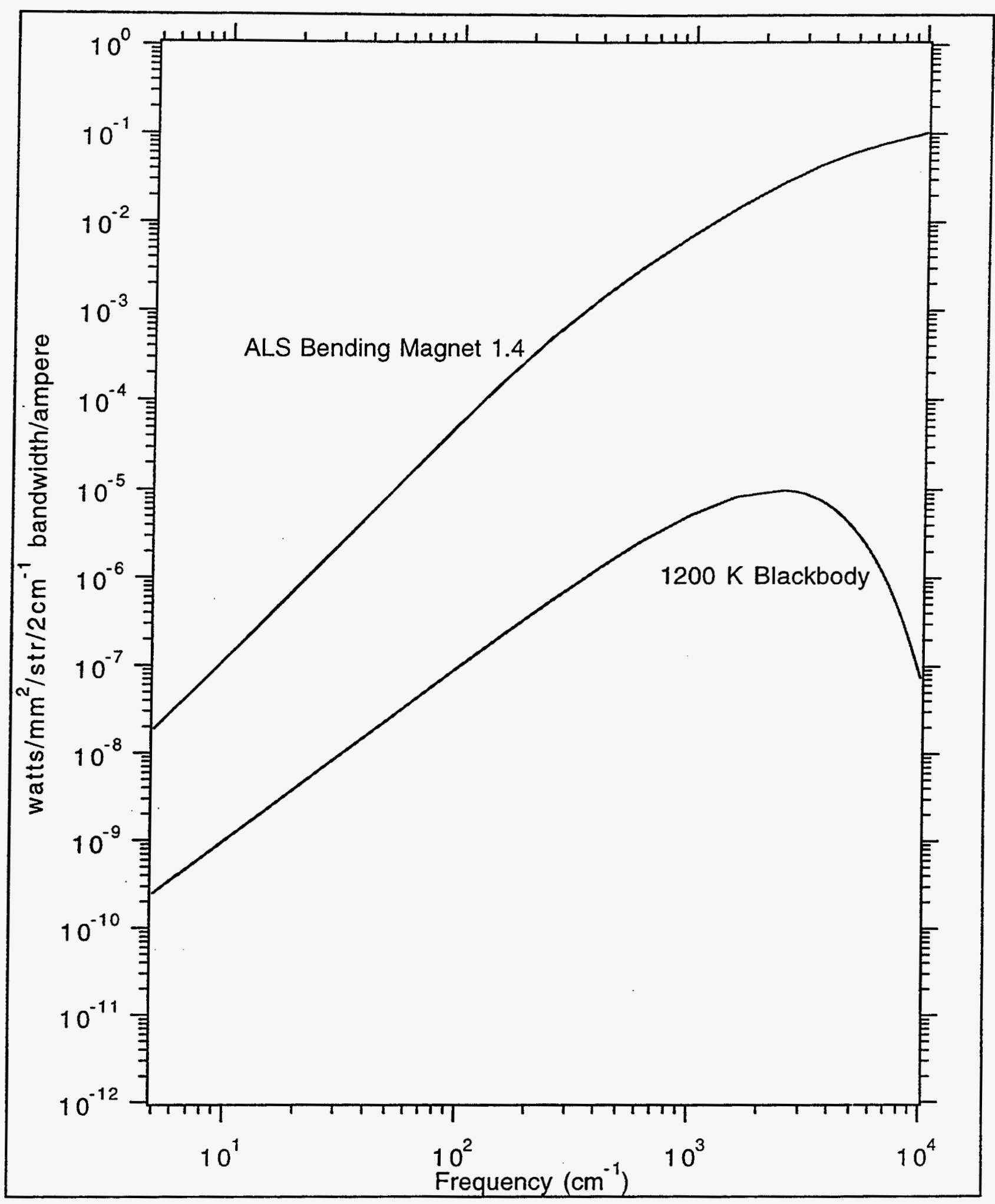

Figure 1: Brightness comparison of ALS bending magnet to blackbody 
This paper will provide a description of the source, the overall layout of the beamline, the optical design considerations (including power handling), and a description of the commissioning of the beamline.

\section{DESCRIPTION OF THE SOURCE}

The beamline uses the $x .4$ port in sector one of the ALS. The beta functions of this port are larger than those of the x. 3 and x.2 ports, making it an ideal port for IR radiation, since the beam sizes are larger than those of the other ports, but do not contribute significantly to the size of the IR source. The one-sigma sizes of the electron beam are $75 \mu$ in the horizontal and $138 \mu$ in the vertical. The spot size of the source can be approximated quite well by adding in quadrature three different effects--the electron beam size, the diffraction size, and the projected size of the emitting region. ${ }^{1}$

The beamline collects $40 \mathrm{mr}$ in the horizontal direction, and $10 \mathrm{mr}$ in the vertical. The geometrical projected size in the horizontal is:

$$
\rho\left(\theta_{\mathrm{h}}{ }^{2}\right) / 8
$$

where $\rho$ is the radius of the bending magnet, and $\theta_{h}$ is the full horizontal angle. The projected size in the vertical can be found by:

$$
\rho \theta_{\mathrm{V}} \theta_{\mathrm{h}} / 8
$$

where $\theta_{\mathrm{v}}$ is the full vertical angle. The size from diffraction broadening is approximated by using $\lambda / \mathrm{d}$. Combining all of these factors gives the following effective source sizes for the wavelength region of interest for microscopy:

\begin{tabular}{|l|l|l|l|l|}
\hline Horizontal & Intrinsic & Projected & Diffraction & Total \\
\hline$\lambda$ in $\mu$ & size in $\mu$ & size in $\mu$ & size in $\mu$ & size in $\mu$ \\
\hline 2 & 75 & 962 & 50 & 966 \\
\hline 10 & 75 & 962 & 250 & 997 \\
\hline 25 & 75 & 962 & 625 & 1150 \\
\hline & & & & \\
\hline Vertical & & & & \\
\hline 2 & 138 & 241 & 200 & 341 \\
\hline 10 & 138 & 241 & 1000 & 1040 \\
\hline 25 & 138 & 241 & 2500 & 2520 \\
\hline
\end{tabular}




\section{OVERall layout}

Figure 2 shows the layout of the beamline. The physical aperture in the storage ring is approximately $10 \mathrm{mr}$ vertical by $80 \mathrm{mr}$ horizontal. Only half of the horizontal aperture can be used, due to interference with a magnet downstream of the bending magnet. A watercooled Glidcop ${ }^{\mathrm{TM}}$ aperture plate absorbs half of the radiation and passes the $10 \mathrm{mr}$ vertical by $40 \mathrm{mr}$ horizontal beam. The aperture plate is followed by a bellows and an all-metal valve which can shut only when the storage ring is not running. (It would quickly overheat if closed during operation.) The first mirror (M1) is a water-cooled mirror of brazed Glidcop ${ }^{\mathrm{TM}}$ with an electroless nickel polished layer. The mirror is located three meters from the tangent point in the bending magnet. The mirror was fabricated at LBNL, and ground and polished by SESO of France. It is novel in that most of it is located outside the UHV chamber, and the mirror body itself forms the seal with the chamber, with an Helico-flex seal. Space considerations drove these choices, and there is considerable advantage to being able to change the mirror for coating and inspection. With proper tenting and dry nitrogen purge, the mirror can be removed during shutdown periods without the need to bake the system after re-installation. The incidence angle on the mirror (M1), 45 degrees, directs the radiation vertically upward onto an ellipsoidal mirror (M2). This is a Zerodur mirror made by conventional techniques, also by SESO. It is placed in the same UHV chamber as M1, and directs the light 90 degrees tangentially to the storage ring and outside of the shielding wall. The 0.5 meter rise will allow future $\mathrm{x}$-ray lines from the previous two bending magnets to pass under the IR beam. A five-inch hole in the shielding wall permits the beam pipe to pass without any lead shielding because of the 0.5 meter vertical difference between the hole and the ring. Measurements show essentially no radiation outside the hole during operation of the ring, and only minimal radiation during injection. The beam passes through a large ion pump just inside the shield wall.

Immediately outside the wall is a large, low vacuum box: the "switchyard." It can be positioned by a six-strut system. Only one rotation is motorized. The UHV extends a short distance into the switchyard. A small, flat mirror turns the radiation towards the microscopy hutch and a diamond window. The diamond window, manufactured by Druker and sold by Harris corporation, is rated to withstand three atmospheres, and is the last UHV component. For additional protection, a small fused-silica-windowed valve with a Viton o-ring seal just before the switchyard shuts automatically if the vacuum fails in the switchyard. The diamond window is $12 \mathrm{~mm}$ in diameter and is placed at the focus of the ellipsoidal mirror. It is sealed in indium foil on both sides, and is polished with a onedegree wedge to remove possible extraneous interference fringes in the FTIR spectra caused by multiple reflections.

The M1/M2 chamber has two motions which can be controlled remotely using DC servomotors. The chamber can be rotated about a vertical axis through the centers of the two mirrors, and the chamber can be moved up and down vertically to center M1 on the height 


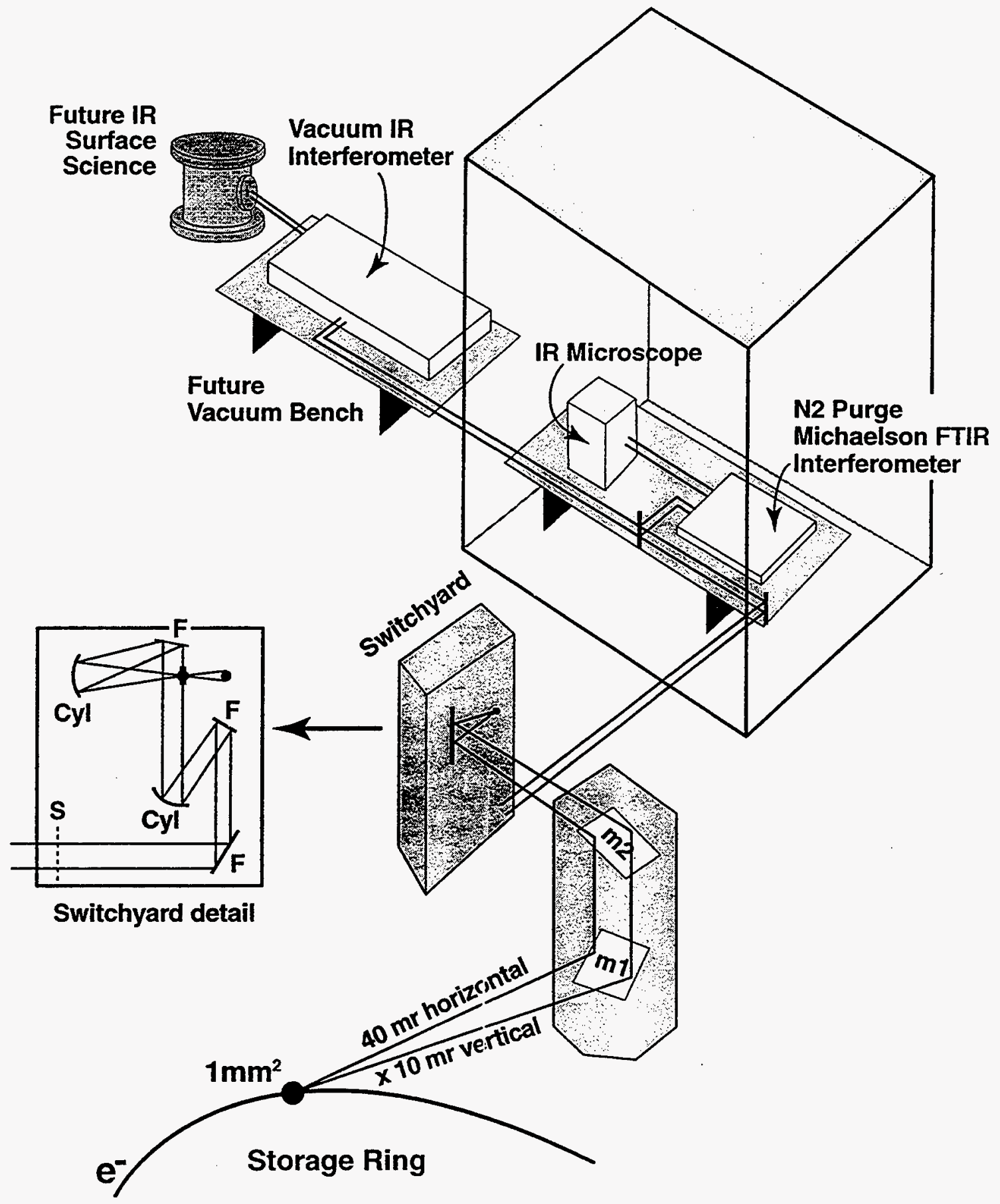

Figure 2: Layout of the IR beamline 1.4 at the ALS 
of the radiated beam. M2 has pitch, roll, and yaw which can be adjusted only from inside the chamber.

Of particular interest is that the beamline has only a minimal equipment protection system (EPS). The M1 mirror, which is the closest mirror to any bending magnet at the ALS, must always be illuminated when the storage ring is running. This minimizes the interaction that the IR user must have with the storage ring, which is of considerable advantage. The components in the UHV area must be robust in order to gain the confidence of the storage ring personnel and other users, since any problems with the IR front end shut down the entire storage ring until the beamline is disconnected and a replacement water-cooled blankoff plate, which absorbs all $80 \mathrm{mr}$ of the radiation, is installed.

Within the switchyard the radiation is allowed to expand four times as far in the $10 \mathrm{mr}$ direction than in the $40 \mathrm{mr}$ direction to allow the beam to be collimated by two separate cylindrical mirrors. This "squares up" the beam to a size which optimally fills one of two microscope objectives of $32 \mathrm{x}$ and $15 \mathrm{x}$. There are two separate collimating/squaring sets of optics which can be moved in and out by motorized control under vacuum. Parallel beams approximately $6 \mathrm{~mm}$ square and $12 \mathrm{~mm}$ square leave the switchyard in the vacuum pipe which leads to the hutch. The cylindrical mirrors are ophthalmic lenses obtained from a local optometrist.

In the hutch, plane mirrors inside the vacuum pipe lead to another wedged diamond window of $20 \mathrm{~mm}$ diameter. The low vacuum stops here and the radiation enters a dry air purged Nicolet 760 FTIR bench. The use of low vacuum all the way to the bench prevents noise from moving purge gas in the long run from the switchyard to the hutch. The bench is followed by a Nic-Plan all-reflective $\mathrm{IR}$ microscope with $\mathrm{LN}_{2}$ cooled detector. The hutch has a small positive pressure from a HEPA filtered air source to keep some degree of cleanliness near the microscope and to provide a secure and isolated environment. Both instruments are supported by an optical table mounted on an interoferometrically stable "six strut" kinematic support system which is successfully in use in throughout the ALS. A hand crank provides rotation of the entire table about the center of the first steering mirror in the transfer pipe at the corner of the table nearest the entrance of the beam, without sacrificing interferometric stability.

For pump-probe timing and IR surface science experiments we have ordered a vacuum FTIR bench from Bruker which will be placed on another table outside the hutch, identical to the one in the hutch which supports the microscope and FTIR bench. The vacuum plumbing will be extended through the hutch wall, onto this table, and into the vacuum instrument. Additional optical components will transfer the beam into an IR surface science experiment. 


\section{Optical Design}

An ALS bending magnet emits about $15 \mathrm{w}$ of radiation per horizontal mr of accepted beam. M1 would normally see $618 \mathrm{w}$ of radiation and would distort too much to be used. To avoid this, a "finger mask" was placed just upstream of M1. The finger mask is an oval copper pipe of approximately 0.140 " height which has approximately one gallon per minute of water running continuously through it. M1 is designed to withstand the entire load, if the beam is mis-steered. In normal operation only 18 watts passes the small finger mask to strike M1. A minimal vertical emitting angle is sacrificed to permit low distortion of the M1 mirror. About 5 watts strikes the $\mathrm{M} 2$ mirror.

The ellipsoidal mirror M2 focuses the SR source at one-to-one magnification at the diamond window in the switchyard. Both of the conjugate distances are specified at 3.5 meters. SESO achieved this specification within a few mm. During alignment the ellipsoid showed excellent imaging performance. All reflecting surfaces are of evaporated aluminum which has been exposed to air. This aluminum oxide top surface layer provides a reflectivity which effectively absorbs radiation in the 10 to 20 volt region which would be passed by gold or silver coatings. Starting after the first diamond window all surfaces in the evacuated transfer pipe are 1-inch diameter flats or cylinders. Figure 3 shows the transmittance of the large diamond window at several locations across the wedged thickness. This transmittance is excellent, and shows only the expected absorption features near $2000 \mathrm{~cm}^{-1}$.

\section{INITIAL COMMISSIONING}

The M1/M2 chamber was connected to the ring during a two-day shutdown. Despite its close location to the ring, the chamber scrubbed in one shift, and the pressure remained within acceptable limits during the entire process. This was aided substantially by the titanium sublimation pump (TSP) at the bottom of the chamber, and by assembly technique (cleanliness). The chamber was centered on the beam by observing the photo-current from the finger mask vary as the chamber was moved vertically while beam was in the ring as shown in Figure 4. The chamber required only 0.007 inch adjustment in the vertical direction to optimize the current from the finger mask. Sophisticated electronics are not needed to read the current. A 100 -ohm resistor to ground provided about 0.3 volt which could be read remotely. Figure 4 shows the variation in beam current with height adjustment of the chamber. It is imperative that this adjustment be done correctly so that significant heat is not allowed to pass onto M1. For additional safety, the cooling water to the finger mask is monitored by thermocouple readouts in both the in-going and outgoing water. This temperature differential did not rise above 2 degrees $\mathrm{F}$, which confirmed the finite element calculations for the finger mask. These calculation indicate that the front edge of the finger mask rises 80 degrees $C$. Careful consideration of stresses and change in properties under thermal cycling is required. 


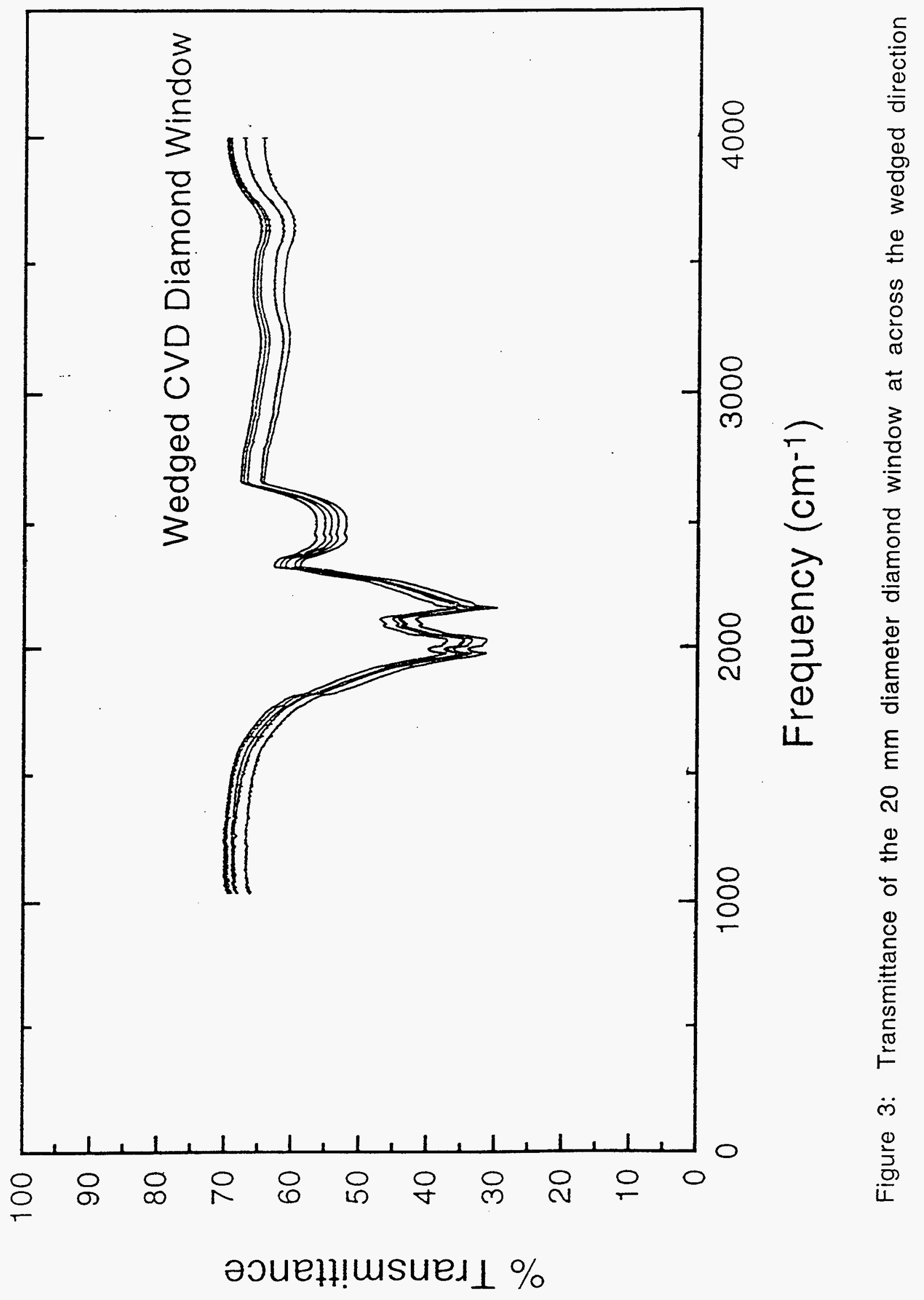


Figure 4: Centering Finger Mask on Beam, July 24, 1997

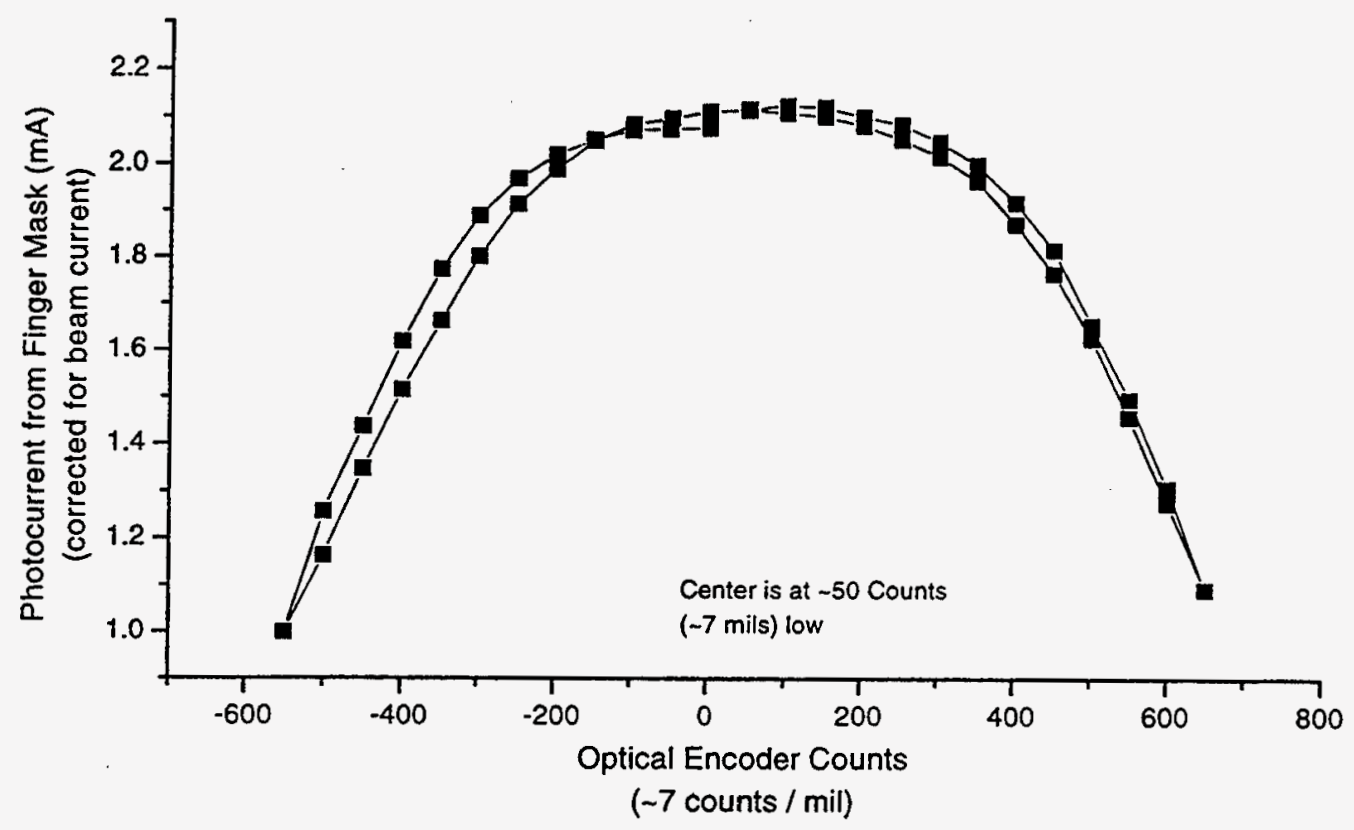

6. SUMMARY

A state-of-the-art beamline for the extraction of IR radiation from a bending magnet at the ALS has been constructed, and is being commissioned. FTIR microscopy at higher spatial resolution than is available in the normal laboratory environment will be available to users. In addition, timing experiments and IR surface science experiments will be able to share the beamline after minimal further construction.

\section{ACKNOWLEDgMENTS}

This work was supported by the Director, Office of Energy Research, Office of Basic Energy Sciences, Materials Sciences Division of the US Department of Energy, under Contract No. DE-AC03-76SF00098. Michael C. Martin assisted in the initial commissioning of the beamline, and provided Figure 4. Greg Vierra drew the layout of the beamline. We also wish to thank Noel Kellogg of the ALS technical staff for expert assembly and commissioning. At all stages of the development, Gwyn Williams and Larry Carr of the NSLS have generously provided the advantages of their extensive experience in IR beamline design and commissioning.

\section{REFERENCES}

${ }^{1}$ Hirschmugl, C.J., PhD Thesis Yale University, 1994. 


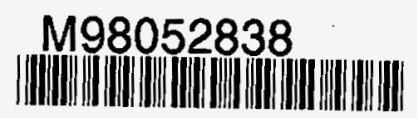

Report Number (14) $\frac{\text { LBNL- }-40848}{\text { CONF-970706- }}$

Jubl. Date (11) 199709

Sponsor Code (18) DOE/ER, XF

JC Category (19) uC-410, DOE/ER 